\title{
Discovery of Therapy-targeting Biomarkers for Post-Concussion Syndrome using Functional Neurocognitive Imaging
}

\section{Caleb T Epps ${ }^{1}$ and Mark D Allen ${ }^{2,3 *}$}

${ }^{1}$ University of North Carolina School of Medicine, Chapel Hill, North Carolina, USA

${ }^{2}$ Cognitive FX, Provo, Utah, USA

${ }^{3}$ Notus Neuropsychological Imaging, Orem, Utah, USA

\begin{abstract}
Background: Post-concussion syndrome (PCS) occurs in a significant percentage of concussion patients. Functional MRI reveals irregular blood-oxygen level dependent signals in PCS patients. PCS biomarkers with functional predictive values have yet to be discovered and validated. Therefore, this study describes five PCS biomarkers and includes a description of their therapeutic application.

Methods: A neurocognitive imaging protocol was developed and a group of healthy control patients was used to generate a normative reference atlas. Biomarker candidate search was performed using an initial sample of PCS patients. Sample validation was applied to each biomarker using a new sample of PCS patients to assess sensitivities/ specificities. A multivariate base rate analysis was performed using 132 new patients and a base rate cutoff matrix was constructed. An example of the biomarker's therapeutic application in a PCS patient is described.

Results: The five functional biomarkers included: Frontal Attentional System hypoactivation, Subcortical System hypoactivation, Visual System hyperactivation, Verbal System hypoactivation, and Frontal/Parietal System hyperactivation. Individual biomarker sensitivities and specificities are reported. Collectively, using the base rate cutoff matrix, a threshold using $3 / 5$ biomarkers below the $10^{\text {th }}$ percentile as the cutoff resulted in a suitable sensitivity $(88 \%)$ and specificity (99\%). The uses of these biomarkers were crucial in guiding the successful treatment of Patient $\mathrm{A}$.

Conclusion: We report the discovery of five functional PCS biomarkers. We show an example of the therapeutic application of the five biomarkers in the successful treatment of PCS. These neuroimaging biomarkers serve to advance diagnostic capabilities and subsequent PCS rehabilitation efforts.
\end{abstract}

Keywords: Post-concussion syndrome; Functional MRI; Biomarker discovery; PCS rehabilitation; Mild traumatic brain injury

\section{Introduction}

Mild traumatic brain injury (mTBI) represents approximately $70 \%$ to $90 \%$ of traumatic brain injuries in the United States with an incidence of 600 in 100,000 people per year [1,2]. Though, the terms mTBI and concussion are often used interchangeably, concussion represents a variety of $\mathrm{mTBI}$ characterized by the absence of structural brain damage, though clinical manifestations may be similar [3]. Concussive symptoms typically resolve in 7 to 10 days (sports-related concussions) or within 3 months (non-athletes) [4]. However, approximately $33 \%$ of patients will have persistence of symptoms with $30 \%$ of those patients meeting post-concussion syndrome (PCS) criteria 6 months out from time of injury [5-8].

Clinically, there are imperfect standards for the diagnosis or treatment of PCS and, despite the given definition, diagnostic criteria are based on subjective symptoms and lack general agreement and specificity. Given this, there are even large differences in the estimated prevalence of PCS. Concerning treatment, PCS patients are traditionally prescribed rest, neurocognitive rehabilitation, education, and antidepressants, all without much evidence of success [9].

Standard structural clinical neuroimaging studies have no abnormal findings for the majority of PCS patients as the clinical presentations of PCS are thought to be caused by cerebrovascular dysregulation and neuronal dysfunction [10-12]. However, functional MRI (fMRI), which uses blood oxygen-level dependent (BOLD) signaling, has shown abnormalities in patients with PCS $[13,14]$. Therefore, fMRI is uniquely poised to serve an essential role in PCS diagnostics and therapeutics. However, fMRI requires two characteristics in order to become relevant in PCS management. First, a normative reference atlas, a reliable and accurate body of data from healthy subjects to which PCS patients can be compared, must be developed. Then second, PCS biomarkers with functional diagnostic predictive values must be discovered and validated.

As there is no wholly reliable standard in the diagnosis and management of PCS [15], the discovery and validation of PCS biomarkers using fMRI would be highly significant and could serve two primary functions. First, not only would reliable PCS biomarkers serve to accurately diagnose PCS, but they would also provide definitive thresholds used by clinicians. This would aid in determining return-toplay for athletes or return-to-work for non-athletes as well as provide data that meet typical standard of evidence criteria in legal settings [15]. Though the importance of this first function should not be understated, the second function of standardized PCS biomarkers would go beyond simply guiding a differential diagnosis. That is, they could serve to guide therapeutic management, as they would help answer not only if PCS pathology is present, but also provide information as to location and

*Corresponding author: Mark D Allen, Notus Neuropsychological Imaging Orem, 280 West River Park Drive, Suite 110, Provo, Utah 84604, USA, Tel: (385) 3758590; Fax: (888) 511-1397; E-mail: notusfmri@gmail.com

Received January 15, 2018; Accepted January 30, 2018; Published February 08, 2018

Citation: Epps CT, Allen MD (2018) Discovery of Therapy-targeting Biomarkers for Post-Concussion Syndrome using Functional Neurocognitive Imaging. Brain Disord Ther 7: 243. doi: 10.4172/2168-975X.1000243

Copyright: $\odot 2018$ Epps CT, et al. This is an open-access article distributed under the terms of the Creative Commons Attribution License, which permits unrestricted use, distribution, and reproduction in any medium, provided the original author and source are credited. 
Citation: Epps CT, Allen MD (2018) Discovery of Therapy-targeting Biomarkers for Post-Concussion Syndrome using Functional Neurocognitive Imaging. Brain Disord Ther 7: 243. doi: 10.4172/2168-975X.1000243

Page 2 of 12

severity of the pathology. In short, dependable PCS biomarkers could serve not only to improve the clinician's diagnostic capabilities, but also, and perhaps of more consequence, to precisely target therapeutic interventions.

Therefore, the present report describes the discovery of five neuroimaging biomarkers in PCS patients using a functional neurocognitive imaging ( $\mathrm{fNCI}$ ) protocol (a standardized form of taskrelated fMRI) and an example of how the biomarkers serve to target interventions in PCS patients. It is hoped that the discovery of these neuroimaging biomarkers might serve to advance both the diagnostic capabilities of clinicians as well as subsequent rehabilitation efforts, thereby improving outcomes for the individual PCS patient.

\section{Methods}

The discovery of therapy-targeting biomarkers for PCS was performed in five stages. First, the neurocognitive imaging protocol used to assess both healthy and PCS patients were developed. Then, a group of healthy control patients $(n=60)$ was used to generate a normative reference atlas to which PCS patients may be compared. A biomarker candidate search was performed using an initial sample of PCS patients $(n=69)$. Independent sample validation was applied to each discovered biomarker using a new sample of PCS patients $(n=120)$ to assess sensitivities and specificities. Finally, because multiple biomarkers were discovered, a multivariate base rate analysis was performed using 132 new patients (62 healthy controls; 70 PCS patients) and a base rate cutoff matrix was constructed. An example of how the biomarkers are used to diagnose and target treatment in a single PCS patient is described.

\section{Ethical approval and informed consent}

This study was approved by the institutional review board and ethics committee of Notus Imaging Research Laboratory (Reference numbers 2015-3, 2016-03, and 2017-03). Prior to participation, each patient signed a consent form giving informed consent. Each patient was informed as to the background of the study, the nature of participation, risks, benefits, how their confidentiality would be protected, and contact information for any additional questions.

\section{Development of the fNCI protocol}

The fNCI assessment protocol combines the validity of conventional neuropsychological testing standards with the reliability and objectivity of informational data output provided by fMRI. The Notus NeuroCogs functional task battery employed in $\mathrm{NCI}$ underwent iterative pilot testing to ensure concurrent validity, reliability, objectivity, and suitability for the MRI scanning environment [16-21], and is comprised of six neuropsychologic test adaptations (Table 1) the functional Matrix Reasoning Test (f-MRT), the functional Trail Making Test-B (f-TMT), the functional Picture Naming Test (f-PNT), the functional Face Memory Test (f-FMT), the functional Verbal Memory Test (f-VMT), and the functional Verbal Fluency Test (f-VFT). Each of the six tasks includes eight test phases presented in alternating fashion with rest phases, in which the subject is asked to silently count from 1 to 10. Compliance monitoring is performed at intervals during each task.

All scanning was performed at the same location using the same standardized administration protocol and functional task battery to limit platform-generated variability. Functional images were acquired with a 1.5-T GE scanner using an EPIBOLD sequence with the critical parameters $\mathrm{TR}=2000 \mathrm{~ms}$; $\mathrm{TE}=40 \mathrm{~ms}$; Flip Angle $=90$. Images were acquired at 23 contiguous axial locations with a slice thickness of 5

\begin{tabular}{|c|c|}
\hline \multicolumn{2}{|r|}{ Notus Neurocogs } \\
\hline $\begin{array}{c}\text { Neuropsychologic } \\
\text { Test }\end{array}$ & Task Description \\
\hline $\begin{array}{l}\text { Functional Matrix } \\
\text { Reasoning Test } \\
\text { (fMRT) }\end{array}$ & $\begin{array}{l}\text { Tests non-verbal problem solving using a } 3 \times 3 \text { array } \\
\text { of visually complex figures with one figure missing. The } \\
\text { subject is instructed to select the best match for the missing } \\
\text { figure from among four "candidate" figures by pressing a } \\
\text { designated button. }\end{array}$ \\
\hline $\begin{array}{l}\text { Functional Trail } \\
\text { Making Test-B } \\
\text { (fTMTB) }\end{array}$ & $\begin{array}{l}\text { Measures cognitive flexibility by presenting virtual connect- } \\
\text { the-dots challenges using a button pad response system. } \\
\text { Randomly arranged numbers and letters are displayed on } \\
\text { a screen and the subject must locate and connect each } \\
\text { series of numbers and letters in ascending order while } \\
\text { alternating back and forth between the two character types. }\end{array}$ \\
\hline $\begin{array}{l}\text { Functional Picture } \\
\text { Naming Test (fPNT) }\end{array}$ & $\begin{array}{l}\text { Assesses semantic object recognition by displaying line } \\
\text { drawings of common objects for a period of } 1.5 \text { seconds } \\
\text { each. Subjects are instructed to silently identify each object } \\
\text { upon presentation. }\end{array}$ \\
\hline $\begin{array}{l}\text { Functional Face } \\
\text { Memory Test } \\
\text { (fFMT) }\end{array}$ & $\begin{array}{l}\text { Assesses long-term memory. Subjects are instructed to } \\
\text { memorize colored photographs of unfamiliar faces and } \\
\text { informed that they will be required to identify some of the } \\
\text { faces at a later time. Twenty faces are presented twice in } \\
2 \text { random orders for three seconds each during scanning. } \\
\text { Recognition accuracy is recorded on a postscan test. }\end{array}$ \\
\hline $\begin{array}{l}\text { Functional Verbal } \\
\text { Memory Test } \\
\text { (fVMT) }\end{array}$ & $\begin{array}{l}\text { Analyzes short-term verbal memory. For each test run, } \\
\text { the subject views a series of eight common words for one } \\
\text { second each and is instructed to silently memorize the } \\
\text { words as they appear. Subjects are given } 12 \text { additional } \\
\text { seconds after all words have been presented to recall as } \\
\text { many as possible. }\end{array}$ \\
\hline $\begin{array}{l}\text { Functional Verbal } \\
\text { Fluency Test (fVFT) }\end{array}$ & $\begin{array}{l}\text { A letter-based fluency test. The subject is instructed } \\
\text { to silently generate as many unique words as possible } \\
\text { (excluding proper names or variants of the same word) } \\
\text { within a } 20 \text {-second time limit using a given first letter. }\end{array}$ \\
\hline
\end{tabular}

Table 1: Notus NeuroCogs functional exams. Each of the six tasks within the Notus NeuroCogs battery is listed with their respective objectives and stimulus descriptions.

$\mathrm{mm}, 0 \mathrm{~mm}$ interslice gap, with a $3.75 \times 3.75 \mathrm{~mm}$ in-plane resolution and a $64 \times 64$ matrix of individual sample points, producing a total of $64 \times 64 \times 23$ voxels for entire brain coverage. Preprocessing procedures included acquisition time realignment, using sinc interpolation, followed by motion correction with echo-planar imaging (EPI) distortion unwarping. No head movement exceeded $1 \mathrm{~mm}$ translation or $1^{\circ}$ rotation displacement. Images were spatially smoothed with an 8-mm FWHM Gaussian kernel. A high-resolution 3D SPGR was co-registered to each individual's mean functional image in order to perform subject-specific functional region analyses that take into account individual variability in cortical landmark organization, for the purposes individual activation extraction requisite for normative reference atlas construction described below.

For each test condition, a time-series analysis of covariance (ANCOVA) implemented in SPM8 was used to test each voxel, for each subject, against the null-hypothesis that changes in BOLD signal in that voxel, over the duration of the experiment, did not significantly correlate with the temporal sequencing of the cognitive task of interest. A boxcar waveform convolved with a synthetic hemodynamic response function (HRF) with a 4-s lag-to-peak was used to model task-related activation. The data were high-passed-filtered in time, using a set of discrete cosine basis functions with a cut-off period of $128 \mathrm{~s}$, and conditioned for temporal autocorrelations using AR1 correction.

In addition to the methods described in here and elsewhere [1621], anatomical regional normalization processes were applied, similar to Voyvodic [22], in order to improve inter-subject reliability and cross platform stability. 
Citation: Epps CT, Allen MD (2018) Discovery of Therapy-targeting Biomarkers for Post-Concussion Syndrome using Functional Neurocognitive Imaging. Brain Disord Ther 7: 243. doi: 10.4172/2168-975X.1000243

\section{Patient demographics}

Demographics for both normative reference volunteers and PCS patients, including inclusion and exclusion criteria, can be found in Table 2.

\section{Development of the normative atlas}

Data analysis revealed 57 specific functional regions (FR) found to be task-associated with each fNCI exam (8-12 FR's per exam).

Methods for deriving normative fMRI data follow a two-stage process. First, statistical activation maps were computed for each subject (as described above), and anatomical boundaries were identified for each subject by a neuroanatomical expert. Second, activation peaks were identified according to an objective algorithm within prespecified anatomical regions (as described in the next paragraph). These pre-specified regions were determined based on prior groupaveraging studies using the similar task-related experimental protocols. Importantly, the normative database in this study is not a result of standard group-averaging techniques typically used in fMRI research. Instead, it is composed of individual data points extracted from individual brain analyses, both in terms of anatomy and activation. This allows the protocols to be acceptably applied at the single-subject level.

The procedure for designating and identifying anatomical region boundaries essentially follows the automated anatomical labeling scheme described by Tzourio-Mazoyer and colleagues [23]. However, it is important to note that as the database was not derived via group averaging, no automated (probabilistic) segmentation was performed. Likewise, no brain space normalization was performed, as is prerequisite for group-averaging procedures. Rather, for this database process, region boundaries were determined in a precise manner for each subject's non-normalized brain. However, each anatomical image

\begin{tabular}{|c|c|}
\hline \multicolumn{2}{|r|}{ Normative Reference Volunteers $(\mathrm{N}=60)$} \\
\hline Demographics & Breakdown \\
\hline Sex & Male $(45 \%)$ : Female $(53 \%)$ \\
\hline Handedness & Right-hand dominance $(91.5 \%)$ \\
\hline Ethnicity & $\begin{array}{l}\text { Caucasian (74.6\%); Hispanic (11.8\%); Asian (10.2\%); African } \\
\text { American (3.4\%) }\end{array}$ \\
\hline First Language & English for all subjects \\
\hline Years of School & $\begin{array}{l}\text { At least one year of higher education for all subjects } \\
\qquad(\text { Mean=14.3, } \sigma=2.9)\end{array}$ \\
\hline \multicolumn{2}{|r|}{ PCS Patients $(\mathrm{N}=259)$} \\
\hline Sex & Male (51\%): Female (48.9\%) \\
\hline Age & Mean $=34.0, \sigma=16.8$ (years) \\
\hline Handedness & $\begin{array}{l}\text { Right-hand dominance }(90.1 \%) \text {; Left-hand dominance }(9.0 \%) \\
\text { Ambidextrous }(0.9 \%)\end{array}$ \\
\hline Ethnicity & $\begin{array}{c}\text { Caucasian (90.8\%); Hispanic (5.5\%); Asian (1.3\%); Pacific } \\
\text { Islander (0.9\%); Native American (0.5\%); Indian (0.5\%); African } \\
\text { American }(0.5 \%)\end{array}$ \\
\hline First Language & English for all subjects \\
\hline Years of School & Mean $=13.7, \sigma=3.4$ \\
\hline Mode of Injury & $\begin{array}{c}\text { Motor Vehicle Accident (37.3\%); Sport-related injury (35.5\%); } \\
\text { Pedestrian/Fall/Other }(27.2 \%)\end{array}$ \\
\hline $\begin{array}{l}\text { Inclusion } \\
\text { Criteria }\end{array}$ & $\begin{array}{l}\text { Any individual diagnosed with mTBI regardless of mode of } \\
\text { or time since injury. All patients on initial trauma met criteria } \\
\text { for mild-TBI and presented with an average Post-Concussion } \\
\text { Symptom Scale index of } 33.5 \text { at the start of treatment. }\end{array}$ \\
\hline
\end{tabular}

Table 2: Healthy control demographics and patient characteristics. The normal healthy control patients were used to develop the normative reference atlas. The PCS patients were used in the neurobiomarkers candidate search $(n=69)$, the independent samples validation $(n=120)$, and the multivariate base rate analysis $(n=70)$. A new sample of healthy volunteers $(n=62)$ was used during the independent samples validation with demographics similar to those used for the normative reference atlas development. Inclusion criteria are provided. was co-registered to the subject's corresponding mean functional image map. Each activation map was also smoothed with a $1.5 \mathrm{~mm}$ FWHM Gaussian spatial filter, in order to condition extreme $t$-value spikes within peak clusters.

After anatomical parcellation, individual functional activation maps (with a single $t$-value assigned to each voxel) were overlain for identification of region peaks. Regions were then inspected for cluster peaks, following these guidelines: If the maximum value within a region belonged to a cluster with a centroid in an adjacent region (i.e., the highest intensity voxel fell at the border of an adjacent region), the region was determined to not have a peak. When more than one peak was identified in a region, the locations of the peaks were catalogued and, if consistently found across subjects ( $>30 \%)$, used to motive further functional region boundary divisions for the cognitive task protocol being analyzed. However, only those regions (or subregions) with peak clusters present in at least $70 \%$ of control subjects were included in the normative data set for each protocol (although in most regions, agreement across subjects in cluster presence/absence exceeded 90\%). Following $t$-value extraction from each subject, means and standard deviations were computed for each region and used to derive normalized $\mathrm{z}$-scores. For each of the six protocols, 8-12 regions met the above requirements for inclusion in the database, for a total of 57 regions. The distributions of $\mathrm{t}$-values in each anatomical region were assessed for normality prior to $\mathrm{z}$-score transformation, on the basis of 60 independent control subjects. Normative reference demographics are outlined in Table 2 below. Anderson-Darling sample-size adjusted tests for normality determined the distributions of each of the 57 regions to be sufficiently normal, with estimates ranging from moderate $\left(\mathrm{A} 2^{*}=0.59, \mathrm{p}=0.11\right)$ to high $\left(\mathrm{A} 2^{*}=0.18, \mathrm{p}=0.91\right)$.

Additionally, these functional regions were found to possess a normal distribution of activation patterns amongst reference subjects. This distributive property formulated a three-dimensional activation standard or normative atlas, which was later used to statistically contextualize both severity and localization of the individual PCS patient activation patterns. An example of this process is outlined in Figure 1. Also, for an example of the assessment of activation patterns in individual patients compared to the normative atlas in all six fNCI exams as studied by Epps et al. [24] (Figures 2 and 3). In order to provide additional reference values for patient analyses below, healthy control subjects were individually evaluated against the group normative data matrix (i.e., a $z$-score was computed from each healthy control data point). The average deviation for reference subjects (mean FR z-scores) was $0.81(\mathrm{SD}=0.20)$.

\section{Biomarker candidate search}

The normative reference atlas makes it possible to search for and verify biomarkers for specific pathologies, that is, reliable patterns of deviation from the norm associated with a specific pathology. Our PCS biomarker development followed a 3-step process: biomarker candidate search, independent samples validation, and multivariate base rate discovery.

Upon development of the normative atlas, the activation patterns in PCS patients undergoing the $\mathrm{ANCI}$ protocol could be evaluated with reference to normal based on location and severity of regional activation deviations. An initial 69 PCS patients underwent the fNCI protocol and z-scores were calculated for each FR in each $\mathrm{ANCI}$ exam. In previous task-related fMRI studies of PCS, distinct patterns of either hyperactivation [25-32] or hypoactivation [26,33-40] have been reliably observed, depending on the cognitive task employed and the cortical 
Citation: Epps CT, Allen MD (2018) Discovery of Therapy-targeting Biomarkers for Post-Concussion Syndrome using Functional Neurocognitive Imaging. Brain Disord Ther 7: 243. doi: 10.4172/2168-975X.1000243

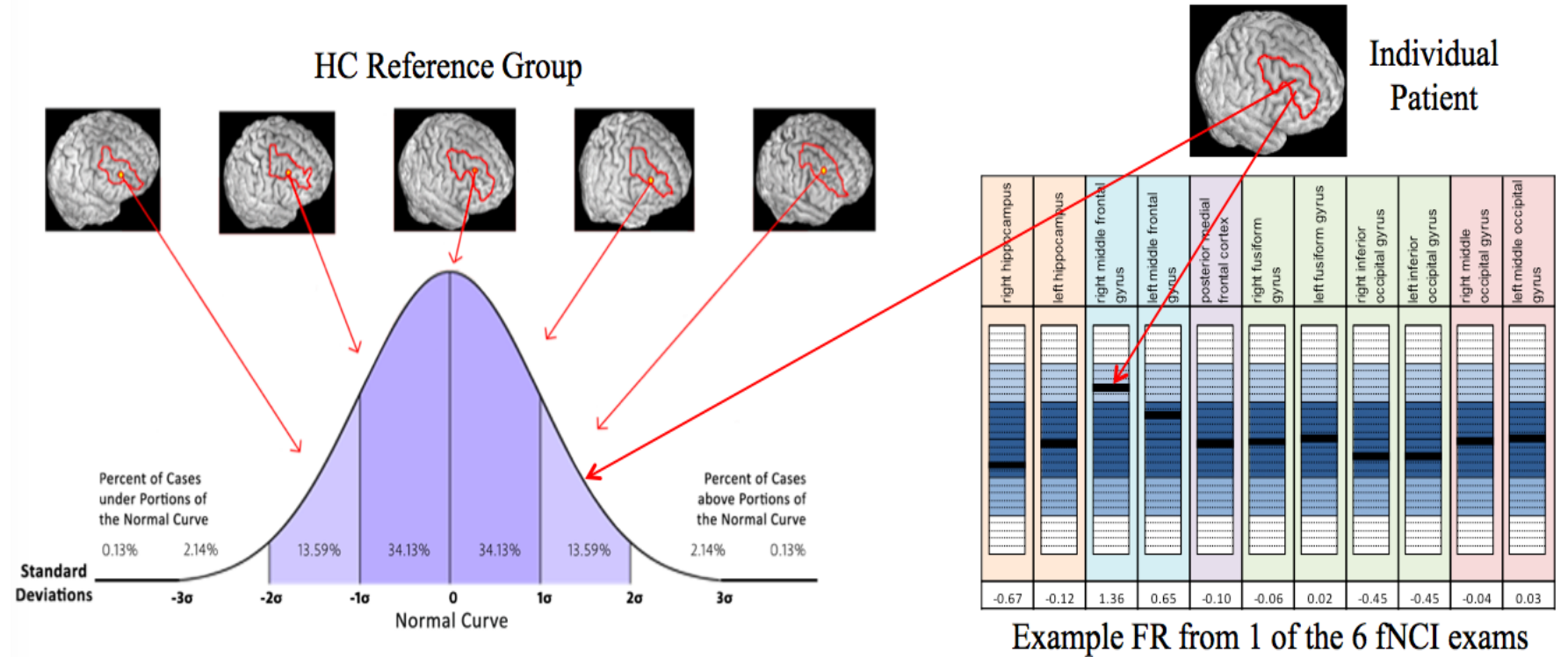

Figure 1: Development of a three-dimensional activation standard per functional region. Data analysis revealed 57 specific neural activation regions, or FR, found to be task-associated with each fNCl exam (8-12 FR per exam). A single data point (Gaussian-smoothed region-normalized activation $t$-score) was extracted from each FR for each subject. All FR were found to possess normally distributed activation data points amongst reference subjects. This analysis resulted in a three-dimensional activation standard or normative atlas, which was later used to statistically contextualize both severity and localization of individual PCS patient activation patterns. Further, analyses of abnormal FR distributions associated specifically with PCS were used to formulate and validate 5 PCS biomarkers. Also, for an example of the contextualization of activation patterns in individual patients compared to the normative atlas in all six fNCl exams, see Epps et al. [22] "Figures 2 \& 3". HC: Healthy Control.

$\mathbf{A}$
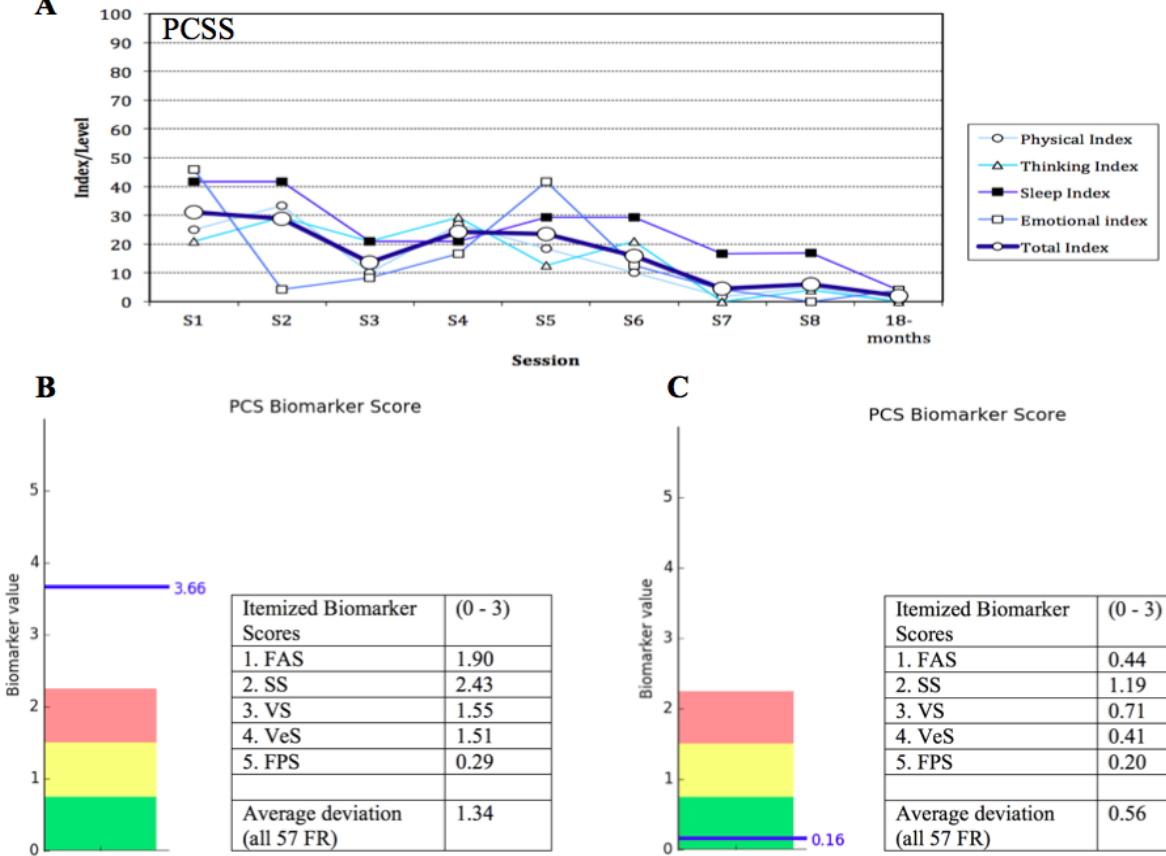

$\mathbf{C}$

PCS Biomarker Score

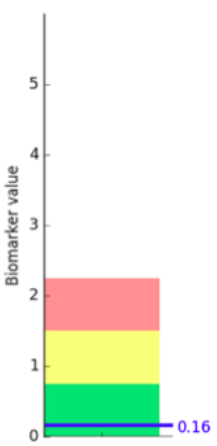

\begin{tabular}{|l|l|}
\hline $\begin{array}{l}\text { Itemized Biomarker } \\
\text { Scores }\end{array}$ & $(0-3)$ \\
\hline 1. FAS & 0.44 \\
\hline 2 . SS & 1.19 \\
\hline $3 . \mathrm{VS}$ & 0.71 \\
\hline 4. VeS & 0.41 \\
\hline 5. FPS & 0.20 \\
\hline & 0.56 \\
\hline $\begin{array}{l}\text { Average deviation } \\
\text { (all 57 FR) }\end{array}$ & 0 \\
\hline
\end{tabular}

Figure 2: Pre- and Post-fNCl and EPIC treatment Post-Concussion Symptom Scale (PCSS), overall SIS, individual biomarker average deviation (z-score), and average deviation across all FR for Patient A. The PCSS (A) is recorded twice daily, at the beginning and end of each EPIC session (4 contiguous days). The PCSS includes 4 subscale indices as seen in the figure key to the right. Each index is made up of a questionnaire investigating symptom exacerbation within each category. The total index (dark blue line) is an average of all indices. PCSS was obtained for Patient A at the time of the follow-up fNCl scan, which was 18 months post-EPIC treatment The SIS is recorded for the pre-treatment $\mathrm{fNCl}$ scan (B; blue line) and post-treatment $\mathrm{fNCl}$ scan (C; blue line). See Methods: Development of Severity Index Score from Discovered Biomarkers for the SIS development methods. The average deviation of each biomarker is displayed as well as the average deviation of all functional regions combined in the tables within panels (B), before treatment, and (C), after treatment. Note, the average deviation for all FR is not the average of the biomarker sub-scores, in that it also includes regions that are not involved in biomarker systems. 
Citation: Epps CT, Allen MD (2018) Discovery of Therapy-targeting Biomarkers for Post-Concussion Syndrome using Functional Neurocognitive Imaging. Brain Disord Ther 7: 243. doi: 10.4172/2168-975X.1000243

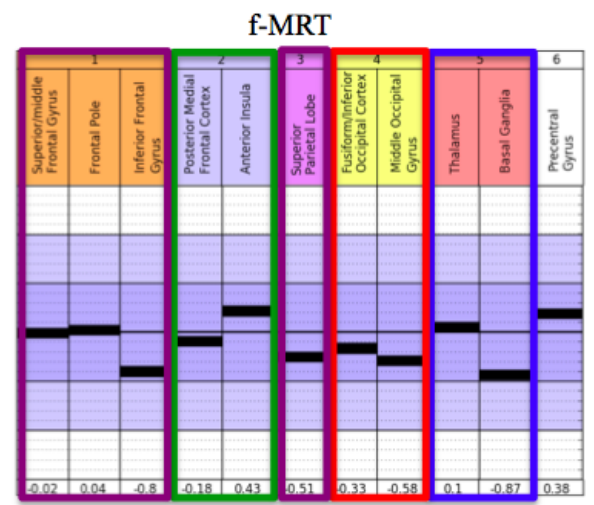

f-FMT

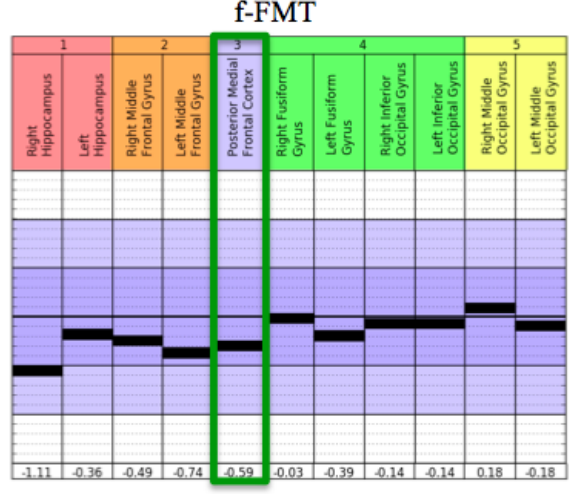

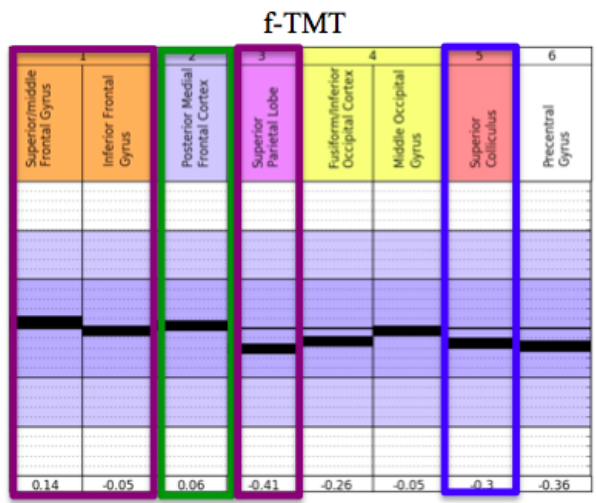

f-VMT

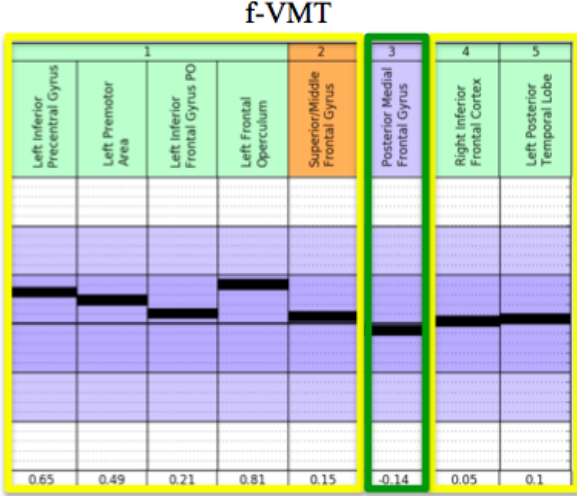

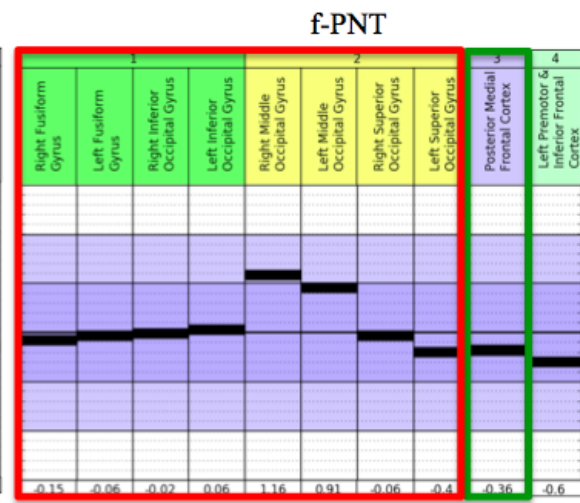

f-VFT

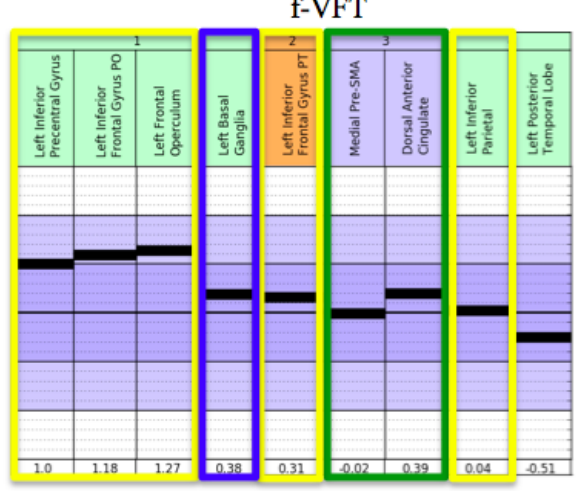

Figure 3: The functional regions involved in five functional neuroimaging PCS biomarkers across all six fNCl exams. The fNCl exams were adapted from classic neuropsychological tests and made suitable for the MRI scanning environment. The exams underwent iterative pilot testing to ensure concurrent validity, reliability, and objectivity. The tests include: the functional Matrix Reasoning Test $(f-M R T)$, the functional Trail Making Test-B $(f-T M T)$, the functional Picture Naming Test ( $f-P N T)$, the functional Face Memory Test (f-FMT), the functional Verbal Memory Test $(f-V M T)$, and the functional Verbal Fluency Test $(f$-VFT). Each fNCl exam was found to have 8-12 associated FR. Prespecified functional-cognitive systems were targeted for analysis. For example, the Frontal Attentional System (FAS) included 1-2 measurements (eight in total) from each $\mathrm{fNCl}$ exam in the medial prefrontal and anterior insular regions. Initial biomarker discovery criteria were then applied to each functional-cognitive system after the functional-cognitive systems analysis was performed. This resulted in the reported five PCS biomarkers involving various FR from the fNCl exams outlined in the following colors: Green (FAS); Blue (SS); Red (VS); Yellow (VeS); Purple (FPS). The example above displays data from a healthy control subject.

region of interest. However, these directional patterns are typically observed across multiple regions, involving coordination between neural systems that are putatively engaged during the execution of the given task at hand. For example, when comparing patients with PCS to healthy controls, working memory tasks (e.g., n-back) have typically shown hyperactivation in dorsolateral prefrontal and parietal cortex $[26,30,41,42]$ and verbal memory tasks have shown hypoactivation in several regions of frontal and temporal cortex in the language dominant hemisphere [33-37]. Accordingly, for biomarker candidate discovery, we grouped functional regions into functional-cognitive systems that would most likely show cohesive levels of either hyper- or hypoactivation in PCS within and across the $6 \mathrm{fNCI}$ tests. For example, the Frontal Attentional System (FAS) included 1-2 measurements (eight in total) from each $\mathrm{ANCI}$ exam in medial prefrontal and anterior insular regions. Initial biomarker discovery criteria were then applied to each candidate functional-cognitive system. These criteria included an average activation $\mathrm{z}$-score $>2$ for all regions in a functional-cognitive system in $>30 \%$ of PCS patients. For example, the FAS was found to be consistently hypoactive ( $\mathrm{z}$-score $>2$ in all regions associated with the FAS) in $>80 \%$ of PCS patients and was therefore considered a biomarker.

\section{Independent samples validation}

Upon identification of the five biomarkers that met the above criteria, independent samples validation was performed. New samples of 120 PCS patients and 52 healthy patients were used to assess the sensitivities and specificities respectively.

\section{Multivariate base rate discovery}

Using independent samples validation, sensitivities and specificities were established for each of the five biomarkers. However, there are unique problems to consider when deriving diagnostic predictive values from multiple biomarkers. That is, each biomarker comes with an inherent false positive rate and if all five biomarkers were applied simultaneously, the individual biomarker's false positive rates would compound one another increasing the potential overall false positive rate. Therefore, we adopted a solution proposed by Iverson et al. [43] where diagnostic values were derived simultaneously from multiple domain scores using multivariate base rate analysis. A uniform cutoff of $+/-2 \mathrm{SD}$ ( $2^{\text {nd }}$ percentile) was used for the initial true positive/false positive rate assessments of each of the five biomarkers. For this initial assessment, five percentile cutoffs were used $\left(2^{\text {nd }}-5^{\text {th }}-10^{\text {th }}-16^{\text {th }}-25^{\text {th }}\right.$ percentiles). Similarly, the successive rates of cumulative biomarkers meeting cutoff criteria at each threshold were assessed (biomarker 1 - biomarker 5). Using new samples of PCS patients $(n=62)$ and healthy controls $(n=70)$, the percent of individuals meeting criteria were calculated for each cumulative-biomarker as well as cutoff-point combination. 
Citation: Epps CT, Allen MD (2018) Discovery of Therapy-targeting Biomarkers for Post-Concussion Syndrome using Functional Neurocognitive Imaging. Brain Disord Ther 7: 243. doi: 10.4172/2168-975X.1000243

Page 6 of 12

\section{Development of severity index score from discovered biomarkers}

The fNCI Severity Index Score (SIS) was developed in order to represent the overall presence of PCS biomarkers in an individual with a single summary score. The score is computed by taking the average activation deviation ( $\mathrm{z}$-score) across all target regions associated with a given biomarker within an individual and multiplying it by the positive predictive value for that biomarker. The SIS then is the sum of this computation for all 5 biomarkers. For all PCS patients, the SIS was found to have a mean of $5.11(\mathrm{SD}=0.89)$, with an approximate range of 3-8. All healthy control subjects were similarly assessed for biomarker severity using the SIS scale, showing an average score of $2.01 \quad(\mathrm{SD}=0.75)$ and approximate range of 1-4 (scores below 1 are nearly impossible with realistic brain activation variability). As the SIS is intended to be used primarily with patients who are independently diagnosed with probable PCS, as opposed to healthy controls, a patient-based SIS scale was developed (the one reported in this study) in which the value 0 was set to the healthy control mean, such that PCS scores tend to fall within the range 0-6. Furthermore, the standard deviation of the SIS from the healthy controls (.75) was used to establish approximate severity range labels (e.g., mild, moderate, severe) for convenience in communicating results with patients and health care practitioners.

\section{Example of biomarker-directed PCS rehabilitation: Patient A}

To demonstrate the use of the five discovered functional neuroimaging biomarkers in targeting PCS therapy, the assessment, management, and results of Patient A's treatment will be described.

Patient A is a previously healthy 25 year-old male who presented to clinic with headaches, low energy, anxiety, dizziness, shaking, fatigue, and "weird sensations and sounds in the head". These symptoms began three years earlier when Patient A hit his head on rock in a riverbed. $\mathrm{He}$ was bedridden for several weeks following the accident and has dealt with the previously mentioned symptoms since this time. This is the second lifetime concussion Patient A has sustained. The first occurred five years prior to presentation during a biking accident in which he sustained head trauma. Following this accident, he began having headaches and weakness sensations but fully recovered after a couple of weeks.

Patient A was referred to clinic for PCS assessment and management. At presentation, his post-concussion symptom scale (PCSS) (total index) inventory was 49 (Figure 2A). He underwent fNCI scanning and both his overall SIS and individual biomarker and FR $\mathrm{z}$-scores were calculated (Figures 2B and 4A).

Across the 6 fNCI exams, Patient A exhibited PCS diagnostic criteria (a FR z-score within biomarker $>2$ ) in $4 / 5$ biomarkers involving $16 \mathrm{FR}$ (average $\mathrm{z}$-score of all $57 \mathrm{FR}=1.34$ ). This included the FAS (average $\mathrm{z}$-score $=1.90$ ), $\mathrm{SS}$ (average $\mathrm{z}$-score $=2.43$ ), VS (average $\mathrm{z}$-score $=1.55$ ), and VeS (average $z$-score=1.52) (see "Results: Biomarker Candidate Search" for abbreviation meanings). The average z-score for the FPS biomarker was within normal range (0.29). His overall SIS was 3.66. Next, based on this data, an individualized treatment plan was formulated. For an overview of the Enhanced Performance in Cognition (EPIC) treatment protocol see Wing et al. [44] section 2.4 "EPIC Treatment". Briefly, the EPIC treatment is a multi-disciplinary protocol involving precisely timed neuromuscular therapy, occupational therapy, athletic training and aerobic exercise, visual rehabilitation using peripheral visual exercises and cognitive therapy including neurocognitive challenges (i.e., brain games). The functional neuroimaging biomarkers guided the multi-disciplinary team's decision as to which aspects of the EPIC treatment would be optimal. For example, Patient's A VS biomarker was abnormal and therefore his treatment included a large peripheral visual exercise component. Another example includes the choice of neurocognitive challenges (i.e., brain games), which factor heavily in the EPIC treatment. In Patient A, the VS biomarker included six hyperactive FR (as seen in the f-PNT test in Figure 4A. therefore, neurocognitive challenges that focused on primary visual processing (basic visual feature processing), complex object recognition (processing complex shapes, forms, and objects), spatial processing (visual-spatial relations, mental imagery, mental object manipulation), and visual search abilities (control of eye movements for visual search) were largely represented in his treatment plan. Further, Patient A also had abnormalities within the VeS biomarker. Therefore, neurocognitive challenges that focused on complex object recognition (processing complex shapes, forms, and objects), left frontal language areas, language and speech motor systems (verbal memory rehearsal, internal speech articulation), word retrieval (transferring words from long term memory into short term memory and retrieving words from long term memory) were included in his treatment plan. On the other hand, the FPS biomarker revealed no abnormalities in Patient A and therefore neurocognitive challenges that focused on executive functioning (planning, reasoning, decision making, cognitive flexibility, executive functions supporting strategic verbal rehearsal, executive functions supporting strategic memory encoding) and motor control (hand/finger control for manual responses) played a less important role in his EPIC treatment.

With an EPIC treatment plan customized according to his functional neuroimaging biomarker abnormalities, Patient A underwent the one-week long treatment. At the end of the week a second, post-treatment fNCI scan was obtained and compared to his pre-treatment scan. Subjectively, his PCSS was followed throughout the week and patterns noted. Both $\mathrm{ANCI}$ and PCSS results are described in "Results" and Figures 2A, 2C and 4B.

\section{Results}

\section{Biomarker candidate search}

As previously mentioned, inclusion criteria for PCS biomarkers included having an average $>2 \mathrm{SD}(+/-)$ for all regions in a functionalcognitive system in $>30 \%$ of patients. Five functional-cognitive systems met these criteria with initial sensitivity rates ranging from $40 \%$ to $90 \%$ as seen in Table 3 and were therefore considered biomarkers. These included: Frontal Attentional System (FAS) hypoactivation, Subcortical System (SS) hypoactivation, Visual System (VS) hyperactivation, Verbal System hypoactivation (VeS), and Frontal/Parietal System

\begin{tabular}{|c|c|c|}
\hline Biomarkers & Sensitivity & Specificity \\
\hline Frontal Attentional System & $88 \%$ & $100 \%$ \\
\hline Subcortical System & $88 \%$ & $100 \%$ \\
\hline Visual System & $79 \%$ & $100 \%$ \\
\hline Verbal System & $65 \%$ & $96 \%$ \\
\hline frontal / Pariental Sytem & $41 \%$ & $99 \%$ \\
\hline
\end{tabular}

Table 3: Sensitivities and specificities of individual functional neuroimaging biomarkers from independent samples validation. Criteria for being considered a PCS biomarker included having an average activation z-score $>2$ for all regions in a functional-cognitive system in $>30 \%$ of PCS patients. For example, the FAS was found to be consistently hypoactive ( $z$-score $>2$ in all regions associated with the FAS) in $>80 \%$ of PCS patients and was therefore considered a biomarker Compared to the initial discovery group, the sensitivities and specificities of the new sample of 120 PCS patients and 52 healthy control subjects were consistent $(+/-5 \%)$. 
Citation: Epps CT, Allen MD (2018) Discovery of Therapy-targeting Biomarkers for Post-Concussion Syndrome using Functional Neurocognitive Imaging. Brain Disord Ther 7: 243. doi: 10.4172/2168-975X.1000243

Page 7 of 12

\begin{tabular}{|c|c|c|c|c|c|}
\hline \multicolumn{6}{|c|}{ Base Rates (in \%) for the $5 \mathrm{f} \mathrm{NCl} \mathrm{Biomarkers} \mathrm{in} \mathrm{Controls} \mathrm{Subjects}$} \\
\hline $\begin{array}{c}\text { Number of Biomarkers } \\
\text { Below Cutoff }\end{array}$ & $\leq 2$ nd Percentile $\mathrm{z}=\mathbf{2 . 0 0}$ & $\leq 5$ th Percentile $\mathrm{z}=1.64$ & $\leq 10$ th Percentile $z=1.28$ & $\leq 16$ th Percentile $z=1.00$ & $\leq 25$ th Percentile $\mathrm{z}=0.67$ \\
\hline 5 & 0 & 0 & 0 & 0 & 0 \\
\hline 4 & 0 & 0 & 0 & 0 & 11 \\
\hline 3 & 0 & 0 & 1 & 12 & 55 \\
\hline 2 & 0 & 0 & 4 & 44 & 78 \\
\hline 1 & 0 & 6 & 23 & 72 & 100 \\
\hline 0 & 100 & 94 & 77 & 28 & 0 \\
\hline
\end{tabular}

Table 4: Multivariate base rate discovery and construction of the base rate cutoff matrix in control patients (false positive rates). As the individual biomarker's false positive rates would compound one another, increasing the potential overall false positive rate, if all five biomarkers were used simultaneously, multivariate base rate analysis was performed and a base rate cutoff matrix was constructed. We adopted a method proposed by Iverson et al. [24] where diagnostic values were derived simultaneously from multiple domain scores using multivariate base rate analysis. A uniform cutoff of $+/-2$ SD $\left(2^{\text {nd }}\right.$ percentile) was used for the initial false positive rate assessments of each of the five biomarkers. For this initial assessment, five percentile cutoffs were used $\left(2^{\text {nd }}-5^{\text {th }}-10^{\text {th }}-16^{\text {th }}-25^{\text {th }}\right.$ percentiles). Similarly, the successive rates of cumulative biomarkers meeting cutoff criteria at each threshold were assessed (biomarker 1 - biomarker 5 ). Using a new sample of healthy controls ( $\mathrm{n}=70$ ), the percent of individuals meeting criteria were calculated for each cumulative-biomarker as well as cutoff-point combination. As an example, using $3 / 5$ biomarkers below the $10^{\text {th }}$ percentile as the cutoff (red highlight), a high specificity $(99 \%)$ is obtained. Therefore, this may serve as a reasonable threshold for both the clinical and non-clinical indications previously discussed.

Base Rates (in \%) for the $5 \mathrm{f} \mathrm{NCl} \mathrm{Biomarkers} \mathrm{in} \mathrm{Controls} \mathrm{Subjects}$

\begin{tabular}{|c|c|c|c|c|c|}
\hline $\begin{array}{c}\text { Number of Biomarkers } \\
\text { Below Cutoff }\end{array}$ & $\leq 2^{\text {nd }}$ Percentile $z=2.00$ & $\leq 5^{\text {th }}$ Percentile $z=1.64$ & $\leq 10^{\text {th }}$ Percentile $z=1.28$ & $\leq 16^{\text {th }}$ Percentile $z=1.00$ & $\leq 25^{\text {th }}$ Percentile $z=0.67$ \\
\hline 5 & 0 & 0 & 4 & 10 & 35 \\
\hline 4 & 0 & 6 & 43 & 61 & 90 \\
\hline 3 & 5 & 20 & 88 & 95 & 100 \\
\hline 2 & 35 & 76 & 100 & 100 & 100 \\
\hline 1 & 89 & 100 & 100 & 100 & 100 \\
\hline 0 & 11 & 0 & 0 & 0 & 0 \\
\hline
\end{tabular}

Table 5: Multivariate base rate discovery and construction of the base rate cutoff matrix in PCS patients (true positive rates). A uniform cutoff of $+/-2$ SD ( $2^{\text {nd }}$ percentile) was used for the initial true positive rate assessments of each of the five biomarkers. For this initial assessment, five percentile cutoffs were used $\left(2^{\text {nd }}-5^{\text {th }}-10^{\text {th }}-16^{\text {th }}\right.$ $-25^{\text {th }}$ percentiles). Similarly, the successive rates of cumulative biomarkers meeting cutoff criteria at each threshold were assessed (biomarker 1 -biomarker 5 ). Using a new sample of PCS patients $(n=62)$, the percent of individuals meeting criteria were calculated for each cumulative-biomarker as well as cutoff-point combination. As an example, using $3 / 5$ biomarkers below the $10^{\text {th }}$ percentile as the cutoff (red highlight), a high sensitivity ( $88 \%$ ) is obtained. Therefore, this may serve as a reasonable threshold for both the clinical and non-clinical indications previously discussed.

(FPS) hyperactivation. An example of how these five biomarkers are contextualized across all $6 \mathrm{fNCI}$ exams in a healthy individual is found in Figure 3.

\section{Independent samples validation}

In the new sample of 120 PCS patients, the true positive rates were consistent with the initial discovery group $(+/-5 \%)$. The sensitivities for the FAS, SS, VS, VeS, and FPS were $88 \%, 88 \%, 79 \%, 65 \%$, and $41 \%$, respectively. The specificities, found using 52 new healthy controls, for each biomarker were $100 \%, 100 \%, 100 \%, 96 \%$, and $98 \%$ respectively. A summary of these results can be found in Table 3 .

\section{Multivariate base rate discovery}

From the multivariate base rate analysis with new samples of healthy controls $(n=62)$ and PCS patients $(n=70)$ as described in the "Methods: Multivariate Base Rate Discovery" section, a base rate cutoff matrix was constructed. Considering the healthy control subjects, using $\leq 2^{\text {nd }}$ percentile $(\mathrm{z}=2.00)$ as the cutoff, $100 \%$ of subjects had $0 / 5$ biomarkers below the cutoff. Using $\leq 5^{\text {th }}$ percentile $(\mathrm{z}=1.64)$ as the cutoff, $94 \%$ of subjects had $0 / 5$ biomarkers below the cutoff and $6 \%$ of subjects had $1 / 5$ biomarkers below the cutoff. Using $\leq 10^{\text {th }}$ percentile $(\mathrm{z}=1.28)$ as the cutoff, $77 \%$ of subjects had $0 / 5$ biomarkers below, $23 \%$ had $1 / 5$ biomarkers below, $4 \%$ had $2 / 5$ biomarkers below, and $1 \%$ had $3 / 5$ biomarkers below the cutoff. Using $\leq 16^{\text {th }}$ percentile $(\mathrm{z}=1.00)$ as the cutoff, $28 \%$ of subjects had $0 / 5$ biomarkers below, $72 \%$ had $1 / 5$ biomarkers below, $44 \%$ had $2 / 5$ biomarkers below, and $12 \%$ had $3 / 5$ biomarkers below the cutoff. Using $\leq 25^{\text {th }}$ percentile $(\mathrm{z}=0.67)$ as the cutoff, $100 \%$ of subjects had $1 / 5$ biomarkers below the cutoff, $78 \%$ had $2 / 5$ biomarkers below, 55\% had 3/5 biomarkers below, and $11 \%$ had
4/5 biomarkers below the cutoff. A summary of these results can be found in Table 4.

Concerning PCS patients, using $\leq 2^{\text {nd }}$ percentile $(\mathrm{z}=2.00)$ as the cutoff, $11 \%$ of patients had 0 biomarkers below the cutoff, $89 \%$ had $1 / 5$ biomarkers below, $35 \%$ had $2 / 5$ biomarkers below, and $5 \%$ had $3 / 5$ biomarkers below the cutoff. Using $\leq 5^{\text {th }}$ percentile $(\mathrm{z}=1.64)$ as the cutoff, $100 \%$ of patients had $1 / 5$ biomarkers below the cutoff, $76 \%$ had $2 / 5$ biomarkers below, $20 \%$ had $3 / 5$ biomarkers below, and $6 \%$ had $4 / 5$ biomarkers below the cutoff. Using $\leq 10^{\text {th }}$ percentile $(\mathrm{z}=1.28)$ as the cutoff, $100 \%$ of patients had at least $2 / 5$ biomarkers below the cutoff, $88 \%$ had $3 / 5$ biomarkers below, $43 \%$ had $4 / 5$ biomarkers below, and $4 \%$ had $5 / 5$ biomarkers below the cutoff. Using $\leq 16^{\text {th }}$ percentile $(\mathrm{z}=1.00)$ as the cutoff, $100 \%$ of patients had at least $2 / 5$ biomarkers below the cutoff, $95 \%$ had $3 / 5$ biomarkers below, $61 \%$ had $4 / 5$ biomarkers below, and $10 \%$ had $5 / 5$ biomarkers below the cutoff. Using $\leq 25^{\text {th }}$ percentile $(\mathrm{z}=0.67)$ as the cutoff, $100 \%$ of patients had at least $3 / 5$ biomarkers below the cutoff, $90 \%$ had $4 / 5$ biomarkers below, and $35 \%$ had $5 / 5$ biomarkers below the cutoff. A summary of these results can be found in Table 5.

The benefit of this analysis is that it provides a transparent means to assess the 'behavior' of multiple biomarkers as a system across a range of cut-off points. This allows for flexibility of criteria depending on the objectives of the biomarkers use. The analysis also reveals consistently high diagnostic values for the 5 biomarkers in aggregate, as demonstrated, for example, at the cut-off point of 3 biomarkers at the $10^{\text {th }}$ percentile, where sensitivity and specificity values are $88 \%$ and $99 \%$, respectively see corresponding red-outlined cells in Tables 4 and 5. However, for the purposes of computing SIS scores, individual positive predictive values were computed for each biomarker using the 
Citation: Epps CT, Allen MD (2018) Discovery of Therapy-targeting Biomarkers for Post-Concussion Syndrome using Functional Neurocognitive Imaging. Brain Disord Ther 7: 243. doi: 10.4172/2168-975X.1000243

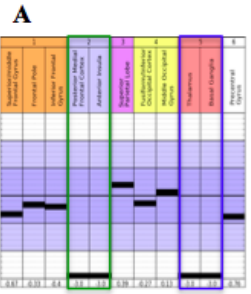

f-MRT

B

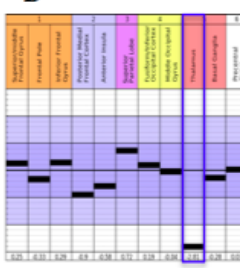

f-MRT

C

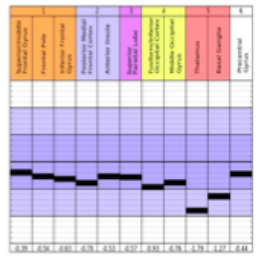

f-MRT

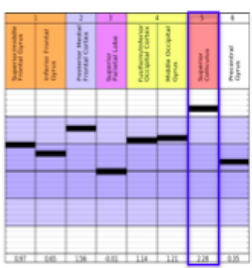

f-TMT

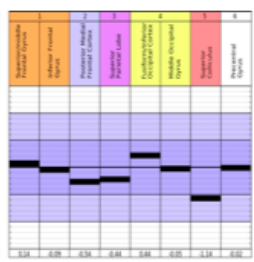

f-TMT

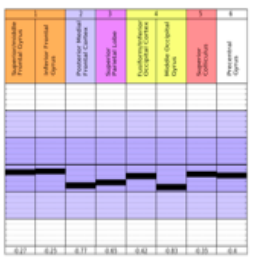

f-TMT

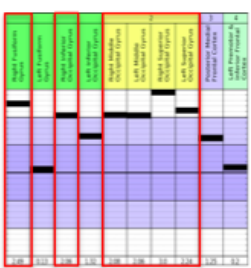

f-PNT

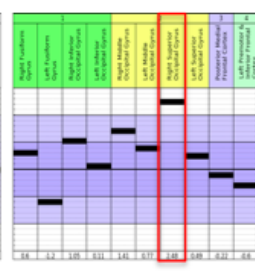

f-PNT

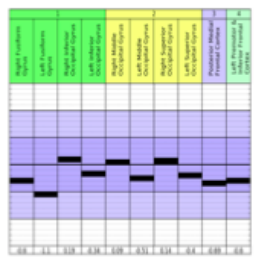

f-PNT

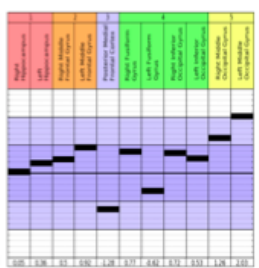

f-FMT

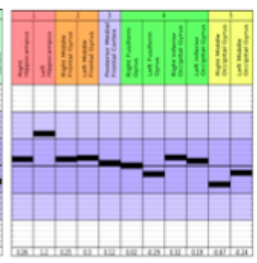

f-FMT

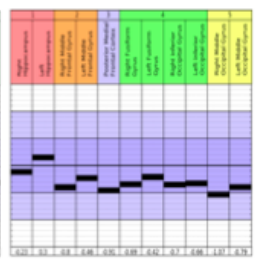

f-FMT

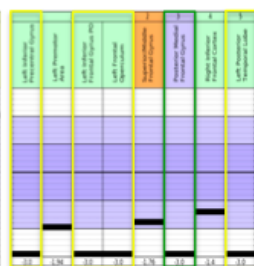

f-VMT

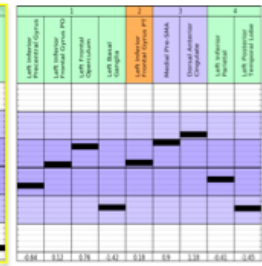

f-VFT

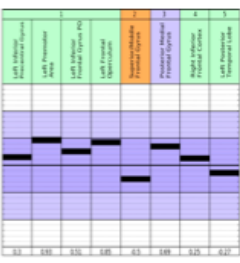

f-VMT

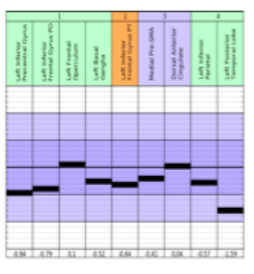

f-VFT

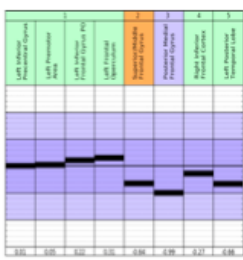

f-VMT

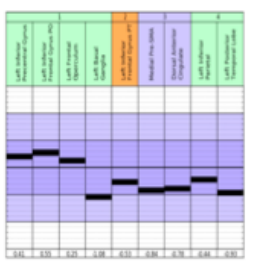

f-VFT

Figure 4: Pre- and post-treatment $\mathrm{fNCl}$ scans for patient $\mathrm{A}$. The z-scores of functional regions involved in PCS biomarkers are contextualized against the normative atlas before (A) and after (B) EPIC treatment. Patient A's pre-treatment $\mathrm{fNCl}$ scan $(\mathbf{A})$ helped to target subsequent neurocognitive rehabilitation as outlined in Methods: Example of Biomarker-Directed PCS Rehabilitation: Patient A. In the pre-treatment $\mathrm{fNCl}$ scan (A), 4/5 abnormal biomarkers involving $16 \mathrm{FR}$ (average Z-score > 2) were observed and outlined in colors assigned to each biomarker (see Figure 3). After EPIC treatment (B), five days later, Patient A exhibited only $2 / 5$ abnormal biomarkers involving $2 \mathrm{FR}$. Panel $\mathrm{C}$ is used to demonstrate the $\mathrm{fNCl}$ scan of Patient $\mathrm{A} 18$-months after treatment and shows normalization of the 2 lingering abnormal $\mathrm{FR}$ in panel

B. For legible $\mathrm{FR}$ names within each $\mathrm{fNCl}$ exam, see Figure 3.

standard sensitivity/specificity values found in "Methods: Independent Samples Validation", in order to provide individually weighted contributions from each biomarker.

\section{Biomarker-directed PCS rehabilitation: Patient A}

Patient A's post-treatment ANCI scan, PCSS, overall SIS, and individual biomarker average $\mathrm{z}$-scores are shown in Figures 2A, 2C and Figure 4B Across the six fNCI exams, Patient A exhibited abnormal activation in $2 / 5$ biomarkers involving only 2 FR (average $\mathrm{z}$-score of all $57 \mathrm{FR}=0.56$ ). The individual average $\mathrm{z}$-scores in all $4 / 5$ biomarkers were significantly decreased compared to pre-treatment scans including the FAS (average $z$-score $=0.44$ ), SS (average $z$-score $=1.19$ ), VS (average $\mathrm{z}$-score $=0.71$ ), and $\mathrm{VeS}$ (average $\mathrm{z}$-score $=0.41$ ). The average $\mathrm{z}$-score for the FPS biomarker continued to fall within the normal range (0.20). His overall SIS was also significantly reduced compared to pre-treatment scan (0.56). Subjectively, his post-treatment PCSS (total index) was also reduced from 49 to 7 . Also of interest, Patient A received an 18-month follow up fNCI scan as reported in Figure 4C. It should be noted that there are further normalizing patterns in FR z-scores found 18-months out from treatment.

Of note, the successful results of 270 PCS patients similar to Patient A who underwent $\mathrm{fNCI}$ scanning and EPIC treatment is reported in Wing et al. [44].

\section{Discussion}

The present report is the first to describe the discovery of five reliable neuroimaging biomarkers using a unique fNCI protocol. The report includes calculations for the sensitivities and specificities of each neuroimaging biomarker and the construction of a base rate cutoff matrix involving all five biomarkers collectively. It is the first to illustrate the use of these neuroimaging biomarkers for both diagnostic and, possibly of more significance, therapy-targeting purposes.

As discussed previously, the goal of neuroimaging biomarker discovery in PCS patients is two-fold. First, these biomarkers provide a significant clinical diagnostic tool. PCS is defined as having a history of traumatic brain injury with persistence of three or more symptoms (i.e., headache, dizziness, fatigue, irritability, insomnia, difficulty in concentration or memory, and intolerance of stress, emotion, or alcohol) [8]. However, because these symptoms are largely subjective and lack specificity, and because often a significant amount of time has passed since the injury, the patient or clinician may not associate the symptoms with a previous mTBI and the underlying PCS pathology is misdiagnosed. Then, for example, the clinician might erroneously treat for depression with antidepressants, or for migraines with sumatriptans and beta-blockers, compounding PCS pathology with medication side effects, further obscuring the true diagnosis. Therefore, the objective neuroimaging biomarkers reported here serve a significant role in guiding differential diagnoses.

Further, these biomarkers serve another diagnostic purpose, providing clinical management and non-clinical thresholds. Examples of clinical management thresholds include: return to play (RTP) for athletes, return to learn (RTL) for students, and return to work for 
Citation: Epps CT, Allen MD (2018) Discovery of Therapy-targeting Biomarkers for Post-Concussion Syndrome using Functional Neurocognitive Imaging. Brain Disord Ther 7: 243. doi: 10.4172/2168-975X.1000243

employees. Non-clinical thresholds include workers compensation for employees injured at work and legal standards regarding TBI-related cognitive impairment for criminal behavior. Considering RTP, current guidelines, according to the Consensus statement on concussion in sport [45], suggest that recovering athletes complete a course of gradually increasing intensity non-contact exercises symptom-free before RTP. The graded protocol includes starting with light aerobic exercise and moving through more intensive training, sports-specific exercises and non-contact participation in the sport, full practice, and finally game-play [45-47]. The athlete must remain symptom free for a minimum of 24 hours at each stage before moving on to the next phase (a minimum of 5 days). Ultimately, a licensed clinical provider who has experience in concussion management should make the final RTP decision.

Successful RTP protocol for student-athletes includes successful RTL. As cognitive recovery often lags behind physical recovery, neuropsychological testing is often used to determine full concussion recovery and RTL. Both pen-and-paper tests and computerized testing have been used to assess patients [48-50]. Pen-and-paper neuropsychological testing can be expensive, time consuming, and interpreter-dependent and as such is often not a practical approach for cognitive recovery management. Computerized neurocognitive assessments take less time and are less expensive. However, because the natural history of concussion is exceedingly individualized, developing generalizable and validated computerized cognitive exams is challenging.

In short, current concussion clinical management protocols lack reliable objective thresholds and continue to be largely based on the subjective symptom experience of the patient. As discussed previously in the "Methods" section, applying multivariate base rate analysis to the five biomarkers, a base rate cutoff matrix was constructed providing options for objective thresholds. For example, using 3/5 biomarkers below the $10^{\text {th }}$ percentile as the cutoff (Tables 4 and 5) a high sensitivity $(88 \%)$ and specificity (99\%) is obtained. Therefore, this may serve as a reasonable threshold for both the clinical and non-clinical indications previously discussed.

The second primary goal of developing neuroimaging biomarkers in PCS is to help target neurocognitive therapies. Compared to the first objective (the use of biomarkers as thresholds), this objective may be more significant when considering PCS specifically. That is, diagnostic thresholds are useful in narrowing the differential diagnosis to PCS. However, if the symptoms persist, which by definition PCS is the persistence of concussion symptoms, then the use of a diagnostic threshold becomes redundant and less functional.

The use of fMRI neuroimaging biomarkers to target therapy is not without precedent. Using a large, multisite sample $(n=1,188)$, Drysdale et al. [51] recently described not only the discovery of functional neuroimaging biomarkers in depression patients but also their use in predicting responsiveness to transcranial magnetic stimulation therapy. Depression serves as a useful model because, like PCS, its clinical presentation is remarkably heterogeneous with many unique possible combinations of symptoms that may co-occur. As such, investigators have been able to describe different forms of depression by identifying clusters of symptoms that tend to co-occur. Further, crude diagnostic biomarkers were then investigated by testing for various neurophysiologic correlates within the clusters. However, these have yet to be proven useful for differentiating depression types in the individual patient or for predicting treatment response of the individual patient. Therefore, Drysdale et al. sought to cluster depression patients according to shared dysfunctional neural signatures on resting state fMRI. They were successful in identifying four depression "biotypes" based on discovered functional neuroimaging biomarkers defined by distinct, homogenous patterns of dysfunctional connectivity. Further, and of particular interest to the present report, they were able to demonstrate the use of these biotypes (defined by functional neuroimaging biomarkers) to predict which patients would have beneficially responsive outcomes with repetitive transcranial magnetic stimulation.

Essentially, having defined functional neuroimaging biomarkers with sensitivities and specificities that are appropriate for diagnostic and therapeutic-guiding uses, both the individual depression patient and the clinician can now answer the question as to which treatment course is right for that individual. Ultimately, the present report seeks a similar aim, which is to demonstrate the therapeutic-guiding capacity of the described five PCS neuroimaging biomarkers.

Figure 4 illustrates the effectiveness of using the five PCS biomarkers in guiding a customized, targeted, and ultimately successful neurocognitive treatment plan. Pre-treatment fNCI scans in Patient A showed 4/5 biomarker abnormalities (z-score $>2$ hyper/ hypoactive) involving 16 FR. Post-treatment scans demonstrated only 2/5 biomarker abnormalities involving 2 FR. However, it should be noted that even though the right superior occipital gyrus FR involved in the VS biomarker remained $>2 \mathrm{z}$-score hyperactive, that is improved from the $>3 \mathrm{z}$-score hyperactivity it demonstrated pre-treatment. The same may be noted for the thalamic FR involved in the SS biomarker, that is, it was reduced from $>3 \mathrm{z}$-score hypoactive pre-treatment to $<3 \mathrm{z}$-score post-treatment. There were similar marked reductions in overall SIS, individual biomarker SIS, and average deviation of all $57 \mathrm{FR}$ when comparing pre-treatment to post-treatment fNCI scans. Further, (Figure 4C) shows stability of biomarker improvement in Patient A 18-months post-treatment and the normalization of the two abnormal post one-week treatment FR. This suggests that the underlying normalized PCS pathology remains stable longitudinally and even continues to improve. Subjectively, all indices of the PCSS (i.e., physical index, thinking index, sleep index, emotional index, and total index) were markedly reduced post-treatment. In short, in both objective and subjective measures, EPIC treatment was regarded as successful for Patient A. The discovered functional biomarkers played a key role in customizing the EPIC treatment to Patient's A specific neurocognitive rehabilitation needs (see "Methods: Discovered Biomarkers Target Therapy for Example Patient A").

It should be noted that the biomarkers in the $\mathrm{ANCI}$ protocol have helped to successfully guide therapy in a similar fashion to Patient A in over 600 PCS patients. The results of 270 of the 600 PCS patients may be found in Wing et al. [44]. Further, 330+ more PCS patients have undergone $\mathrm{fNCI}$ imaging and EPIC treatment since this publication, with similarly successful results.

As technologies in human neuroimaging continue to advance, specifically in the fMRI domain, biomarkers will play increasingly important roles in disease diagnostics and therapeutics. Concerning the development of biomarkers, Woo et al. [52] describe a set of desirable characteristics that functional biomarkers should display. The first phase of biomarker development is the discovery stage. Within this stage, the biomarker should demonstrate both diagnosticity (Criterion 1) and interpretability (Criterion 2). The diagnostic value may be evaluated by sensitivity and specificity. Sensitivity deals with whether the model can accurately identify signal when signal exists and specificity deals with whether the model does not detect signal 
Citation: Epps CT, Allen MD (2018) Discovery of Therapy-targeting Biomarkers for Post-Concussion Syndrome using Functional Neurocognitive Imaging. Brain Disord Ther 7: 243. doi: 10.4172/2168-975X.1000243

when no signal is present. Interpretability refers to the capability of the biomarker to be meaningful in terms of any prior neuroimaging studies or evidence from other sources. That is, the biomarker should avoid capitalizing on confounding variables that are not neuro-scientifically meaningful, such as scanner head movement [53]. After discovery, the second phase of biomarker development is validation. This includes deployability (Criterion 3 ) and generalizability (Criterion 4). Deployability refers to precisely defining the protocol used to identify the biomarker so that it can be reliably and predictably applied to new data. A standardized testing procedure is critical so that the biomarker can be shared, tested, and further validated across various labs. With criterion 3 fulfilled, the generalizability of the biomarker can be tested. That is, the performance of the standardized testing procedure should be prospectively tested across different labs, scanners, populations, and indications thereby validating its use.

Though it is believed that the described five biomarkers largely fulfill the desired characteristics set out by Woo et al., it should be noted that the goals of such characteristics seem to be more diagnostic in nature than therapy-targeting. A primary objective of discovering the reported biomarkers is to help guide neurocognitive therapy in PCS patients as laid out in the example of Patient A. Therefore, they should demonstrate characteristics other than the four previously mentioned criteria. That is, therapy-targeting biomarkers should demonstrate not only the ability to identify PCS pathology location and severity, but also the ability to predict which treatments would be most beneficial in treating the pathology. They should also demonstrate the capacity to monitor treatment efficacy. As demonstrated in Patient A, the reported five biomarkers fulfill these therapy-guiding characteristics.

It should be noted that the reported biomarkers are derived from task-related functional neuroimaging as opposed to resting state functional MRI (rsfMRI). Several studies have investigated rsfMRI abnormal signal patterns in concussion [54-60]. For example, Zhu et al. [54] conducted a pilot study in which they used 8 concussed divisions I collegiate football players and 11 control subjects to search for biomarkers within the default mode network (DMN) longitudinally. Specifically, they obtained diffusion-tensor and rsfMRI images in patients at 24 hours, 7 days, and 30 days post-concussion. Network based and whole-brain based functional correlation analyses on the DMN were performed comparing concussed patients to healthy controls. They found, compared to the control group, a general trend of increased DMN connectivity at 24 hours, a drop in DMN connectivity at day 7 , and then partial recovery of the DMN functional connectivity at day 30. They suggest this biomarker can be used "to monitor the dynamically changing brain function after sports-related concussion". That is, it primarily serves a diagnostic and treatment monitoring, not targeting, role. A similar theme is found in other studies using rsfMRI in concussion [54-60].

In other words, there is limited data regarding the relationship between active intervention in concussion rehabilitation and the ability to restore resting state functional connectivity networks. However, as Wing et al. [44] and the present report demonstrate, the EPIC treatment for PCS appears to actively rehabilitate cognitive task-related, coordinated functional systems that correlate with successful clinical and subjective patient outcomes. Therefore, it stands to reason that biomarkers used in PCS should be derived from taskrelated functional imaging so as to target pathologic, cognitive taskrelated functional regions. Further, a significant subset of PCS patients largely experience symptomatic exacerbation upon cognitive task initiation and are mostly symptom free at rest. Task-related functional neuroimaging biomarkers would cover this subset of patients. In sum, the task-related protocol (fNCI) used to derive biomarkers in this study was designed from the outset as an assessment battery for neurocognitive performance. The advantage of this form of imaging is that it may detect more subtle forms of pathology, such as PCS, that may only emerge when the system is pushed during the demands of actual cognitive challenge, and/or when it must coordinate transient activity between functional regions for the performance of a given task, that may lack the sort of inherent "resting-state" connections observed in functional connectivity imaging approaches.

\section{Limitations}

In terms of limitations, considering the optimal characteristics of neuroimaging biomarkers laid out by Woo et al. [52], it is largely felt that the discovery of the reported five biomarkers meet the four criteria. However, concerning criterion 4, "Generalizability", the argument is less compelling than the first three. According to Woo et al. [52], clinically useful neuroimaging biomarkers should have their performance proved across different laboratories, scanners, protocols, populations, and testing conditions. Although methods were employed to improve cross-site imaging stability [22], the fNCI protocol used to discover the five neuroimaging biomarkers is largely confined to the clinic in which the research occurred. Also, because the EPIC treatment protocol is multi-disciplinary involving precisely coordinated efforts from neuromuscular therapy, occupational therapy, athletic training, neurocognitive therapy, and visual therapy, it may be complicated to easily replicate in other research teams. On the other hand, it is felt that the fNCI protocol used to identify the functional neuroimaging biomarkers is precisely defined and the testing procedure used in the protocol standardized, increasing the generalizability potential. Further, a main objective of the discovery of these neuroimaging biomarkers is the guidance of therapeutic interventions. That is, the validation of their usefulness in targeting PCS interventions within the EPIC protocol was an essential characteristic when searching for PCS functional neuroimaging biomarkers in the present study. As demonstrated in example Patient A, and in 270 PCS patients reported in Wing et al. [44], the biomarkers appear to fulfill this essential characteristics.

\section{Conclusions and Future Directions}

In summary, the reported five functional neuroimaging biomarkers have significant diagnostic and therapy-guiding implications. Specifically, diagnostic uses include guiding differential diagnoses and providing clinical and non-clinical thresholds (RTP, RTL, workers compensation, etc.). Therapy-targeting implications are outlined in the example of Patient A as well as the 270 PCS patients reported in Wing et al. [44]. As discussed, the task-based patterns of hypo-/hyperactivation demonstrated found in the biomarkers is highly consistent with the current corpus of task-related fMRI findings in PCS. It is felt that the reported biomarkers largely fulfill the criteria of successful neuroimaging biomarkers as described in Woo et al. [52]. Lastly, limitations of this study include a potential lack in generalizability of the biomarkers.

In conclusion, the discovery of five functional neuroimaging biomarkers in PCS patients, specifically PCS therapy-targeting biomarkers, is outlined in the present report. Also reported are calculations for the sensitivities and specificities of each biomarker individually as well as a base rate cutoff matrix involving all five biomarkers collectively. The report further outlines an example, in 
Citation: Epps CT, Allen MD (2018) Discovery of Therapy-targeting Biomarkers for Post-Concussion Syndrome using Functional Neurocognitive Imaging. Brain Disord Ther 7: 243. doi: 10.4172/2168-975X.1000243

Patient A, of the therapeutic application of the five biomarkers in the successful treatment of PCS. Results of PCS treatment success in 270 patients is reported in Wing et al. [44]. Future studies may include a) the application of the fNCI protocol to search for reliable neuroimaging biomarkers in other neurocognitive disorders, such as mild cognitive impairment, b) the use of the reported biomarkers in the acute concussion setting, c) head-to-head comparison of current $\mathrm{RTP} / \mathrm{RTL}$ protocols and the reported biomarkers in successful RTP/ RTL in athletes and students, and d) the reproducibility of the $\mathrm{NCI}$ protocol in other research and clinical environments.

\section{References}

1. Cassidy JD, Carroll LJ, Peloso PM, Borg J, Von Holst H, et al. (2004) Incidence, risk factors and prevention of mild traumatic brain injury: results of the WHO Collaborating Centre Task Force on Mild Traumatic Brain Injury. J Rehabil Med pp: $28-60$

2. Faul M XL, Wald MM, Coronado V (2010) Traumatic brain injury in the United States: Emergency department visits, hospitalizations and deaths, 2002-2006. Atlanta.

3. McCrory P, Meeuwisse WH, Aubry M, Cantu B, Dvorak J, et al. (2013) Consensus statement on concussion in sport: the 4th International Conference on Concussion in Sport held in Zurich, November 2012. Br J Sports Med 47 250-258.

4. McCrea M, Guskiewicz KM, Marshall SW, Barr W, Randolph C, et al. (2003) Acute effects and recovery time following concussion in collegiate footbal players: the NCAA Concussion Study. JAMA 290: 2556-2563.

5. Mittenberg W, Canyock EM, Condit D, Patton C (2001) Treatment of postconcussion syndrome following mild head injury. J Clin Exp Neuropsychol 23: 829-836

6. Rimel RW, Giordani B, Barth JT, Boll TJ, Jane JA (1981) Disability caused by minor head injury. Neurosurgery 9: 221-228.

7. Binder LM, Rohling ML, Larrabee GJ (1997) A review of mild head trauma. Part I: Meta-analytic review of neuropsychological studies. J Clin Exp Neuropsychol 19: 421-431.

8. World Health Organization (1993) International Statistical Classification of Diseases and Related Health Problems (10th edn) World Health Organization, Geneva, Switzerland pp: 63-64.

9. Leddy JJ, Kozlowski K, Fung M, Pendergast DR, Willer B (2007) Regulatory and autoregulatory physiological dysfunction as a primary characteristic of post concussion syndrome: Implications for treatment. NeuroRehabilitation 22: 199205

10. Giza CC, Hovda DA (2001) The neurometabolic cascade of concussion. J Ath Train 36: 228-235

11. Ellis MJ, Leiter J, Hall T, McDonald PJ, Sawyer S, et al. (2015) Neuroimaging findings in pediatric sports-related concussion. J Neurosurg Pediatr 16: 241 247.

12. Giza CC, Hovda DA (2014) The new neurometabolic cascade of concussion Neurosurgery 75 Suppl 4: S24-33

13. Eierud C, Craddock RC, Fletcher S, Aulakh M (2014) Neuroimaging after mild traumatic brain injury: review and meta-analysis. Neuroimage Clin 4: 283-294.

14. Dean PJ, Sato JR, Vieira G, McNamara A, Sterr A (2015) Multimodal imaging of mild traumatic brain injury and persistent post-concussion syndrome. Brain Behav 5: 45-61.

15. McCrory P, Meeuwisse W, Aubry M, Cantu B, Dvorak J, et al. (2013) Consensus statement on concussion in sport--the 4th International Conference on Concussion in Sport held in Zurich, November 2012. Clin J Sport Med 23 . 89-117.

16. Allen MD, Fong AK (2008) Clinical application of standardized cognitive assessment using fMRI. I. Matrix reasoning. Behav Neurol 20: 127-140.

17. Allen MD, Fong AK (2008) Clinical application of standardized cognitive assessment using fMRI. II. Verbal fluency. Behav Neurol 20: 141-152.

18. Allen MD, Hedges DW, Farrer TJ, Larson MJ (2012) Assessment of brain activity during memory encoding in a narcolepsy patient on and off modafinil using normative fMRI data. Neurocase 18: 13-25.
19. Allen MD, Owens TE, Fong AK, Richards DR (2011) A functional neuroimaging analysis of the Trail Making Test-B: implications for clinical application. Behav Neurol 24: 159-171.

20. Woon FL, Allen MD, Miller CH, Hedges DW (2012) The functional magnetic resonance imaging-based verbal fluency test in obsessive-compulsive disorder. Neurocase 18: 424-440.

21. Allen MD WT, Bigler ED (2011) Traumatic brain injury alters word memory test performance by slowing response time and increasing cortical activation: An fMRI Study of a Symptom Validity Test. . Psychol Inj Law 4: 140-146

22. Voyvodic JT (2012) Reproducibility of single-subject fMRI language mapping with AMPLE normalization. J Magn Reson Imaging 36: 569-580.

23. Tzourio-Mazoyer N, Landeau B, Papathanassiou D, Crivello F, Etard O et al. (2002) Automated anatomical labeling of activations in SPM using a macroscopic anatomical parcellation of the MNI MRI single-subject brain. Neuroimage 15: 273-289.

24. Epps CT TB, Fong AK, Allen MD (2017) Functional NeuroCognitive Imaging ${ }^{\mathrm{TM}}$ and Notus NeuroCogs ${ }^{\mathrm{TM}}$ : A novel use of fMRI in the assessment of cognitive function in major neurocognitive disorder. J Neuroimaging Psychiatry Neurol 2(1): 20-29.

25. Allen MD WT, Bigler ED (2011) Traumatic brain injury alters word memory test performance by slowing response time and increasing cortical activation: An fMRI Study of a Symptom Validity Test. Psychological Injury and Law 4: 140146.

26. McAllister TW SM, Flashman LA, Guerin SJ, Ford JC, Mamourian AC et al (2001) Reduction in episodic memory fMRI circuitry activation is related to traumatic brain injury severity. The Journal of Neuropsychiatry and Clinical Neurosciences 13: 141

27. Slobounov SM, Zhang K, Pennell D, Ray W, Johnson B, et al. (2010) Functional abnormalities in normally appearing athletes following mild traumatic brain injury: A functional MRI study. Exp Brain Res 202: 341-354.

28. Jantzen KJ, Anderson B, Steinberg FL, Kelso JA (2004) A prospective functional MR imaging study of mild traumatic brain injury in college footbal players. AJNR Am J Neuroradiol 25: 738-745.

29. Lovell MR, Pardini JE, Welling J, Collins MW, Bakal J, et al. (2007) Functiona brain abnormalities are related to clinical recovery and time to return-to-play in athletes. Neurosurgery 61: 352-359

30. McAllister TW, Saykin AJ, Flashman LA, Sparling MB, Johnson SC, et al. (1999) Brain activation during working memory 1 month after mild traumatic brain injury: A functional MRI study. Neurology 53: 1300-1308.

31. Scheibel RS, Newsome MR, Troyanskaya M, Lin X, Steinberg JL, et al. (2012) Altered brain activation in military personnel with one or more traumatic brain injuries following blast. J Int Neuropsychol Soc 18: 89-100.

32. Smits M, Dippel DW, Houston GC, Wielopolski PA, Koudstaal PJ, et al. (2009) Post-concussion syndrome after minor head injury: Brain activation of working memory and attention. Hum Brain Mapp 30: 2789-2803.

33. Chen JK, Johnston KM, Collie A, McCrory P, Ptito A (2007) A validation of the post concussion symptom scale in the assessment of complex concussion using cognitive testing and functional MRI. J Neurol Neurosurg Psychiatry 78: 1231-1238.

34. Chen JK, Johnston KM, Frey S, Petrides M, Worsley K, et al. (2004) Functional abnormalities in symptomatic concussed athletes: an fMRI study. Neuroimage 22: 68-82.

35. Chen JK, Johnston KM, Petrides M, Ptito A (2008) Recovery from mild head injury in sports: evidence from serial functional magnetic resonance imaging studies in male athletes. Clin J Sport Med 18: 241-247.

36. Chen JK JK, Collie A, McCrory P, Ptito A (2004) Association between symptom severity, cogsport tests results, and functional MRI activation in symptomatic concussed athletes. Clinical Journal of Sport Medicine 14: 379.

37. Chen JK, Collie A, McCrory P, Ptito A (2004) Behavioural and functional imaging outcomes in symptomatic concussed athletes measured with cogsport and functional MRI. Br J Sports Med 38: 659.

38. Mayer AR, Mannell MV, Ling J, Elgie R, Gasparovic C, et al. (2009) Auditory orienting and inhibition of return in mild traumatic brain injury: A FMRI study. Hum Brain Mapp 30: 4152-4166.

39. Matthews S, Simmons A, Strigo I (2011) The effects of loss versus alteration 
Citation: Epps CT, Allen MD (2018) Discovery of Therapy-targeting Biomarkers for Post-Concussion Syndrome using Functional Neurocognitive Imaging. Brain Disord Ther 7: 243. doi: 10.4172/2168-975X.1000243

of consciousness on inhibition-related brain activity among individuals with a history of blast-related concussion. Psychiatry Res 191: 76-79.

40. Stulemeijer M, Vos PE, Van der Werf S, Van Dijk G, Rijpkema M, et al. (2010) How mild traumatic brain injury may affect declarative memory performance in the post-acute stage. J Neurotrauma 27: 1585-1595.

41. McAllister TW, Flashman LA, McDonald BC, Saykin AJ (2006) Mechanisms of working memory dysfunction after mild and moderate TBI: evidence from functional MRI and neurogenetics. J Neurotrauma 23: 1450-1467.

42. McAllister TW, Sparling MB, Flashman LA, Guerin SJ, Mamourian AC, et al. (2001) Differential working memory load effects after mild traumatic brain injury. Neuroimage 14: 1004-1012.

43. Iverson GL, Brooks BL, Langenecker SA, Young AH (2011) Identifying a cognitive impairment subgroup in adults with mood disorders. J Affect Disord 132: 360-367.

44. Wing BH TB, Fong AK, Allen MD (2017) Developing the standard of care for post-concussion treatment: Neuroimaging-guided rehabilitation of neurovascular coupling. The Open Neuroimaging Journal 11: 3-16.

45. McCrory P, Meeuwisse W, Dvorak J, Aubry M, Bailes J, et al. (2017) Consensus statement on concussion in sport-the 5th international conference on concussion in sport held in Berlin, October 2016. Br J Sports Med 51: 838847

46. Halstead ME, Walter KD (2010) Council on Sports M: Fitness American Academy of Pediatrics. Clinical report-sport-related concussion in children and adolescents. Pediatrics 126: 597-615.

47. Harmon KG, Drezner J, Gammons M, Guskiewicz K, Halstead M, et al. (2013) American Medical Society for Sports Medicine position statement: concussion in sport. Clin J Sport Med 23: 1-18.

48. Van Kampen DA, Lovell MR, Pardini JE, Collins MW, Fu FH (2006) The "value added" of neurocognitive testing after sports-related concussion. Am J Sports Med 34: 1630-1635.

49. Broglio SP, Macciocchi SN, Ferrara MS (2007) Neurocognitive performance of concussed athletes when symptom free. J Athl Train 42: 504-508.
50. Makdissi M, Darby D, Maruff P, Ugoni A, Brukner P, et al. (2010) Natural history of concussion in sport: markers of severity and implications for management Am J Sports Med 38: 464-471.

51. Drysdale AT, Grosenick L, Downar J, Dunlop K, Mansouri F, et al. (2017) Resting-state connectivity biomarkers define neurophysiological subtypes of depression. Nat Med 23: 28-38.

52. Woo CW, Wager TD (2015) Neuroimaging-based biomarker discovery and validation. Pain 156: 1379-1381.

53. Eloyan A, Muschelli J, Nebel MB, Liu H, Han F, et al. (2012) Automated diagnoses of attention deficit hyperactive disorder using magnetic resonance imaging. Front Syst Neurosci 6: 61

54. Zhu DC, Covassin T, Nogle S, Doyle S, Russell D, et al. (2015) A potential biomarker in sports-related concussion: brain functional connectivity alteration of the default-mode network measured with longitudinal resting-state fMRI over thirty days. J Neurotrauma 32: 327-341.

55. Johnson B, Zhang K, Gay M, Horovitz S, Hallett M, et al. (2012) Alteration of brain default network in subacute phase of injury in concussed individuals: Resting-state fMRI study. Neuroimage 59: 511-518.

56. Slobounov SM, Gay M, Zhang K, Johnson B, Pennell D, et al. (2011) Alteration of brain functional network at rest and in response to YMCA physical stress test in concussed athletes: RsFMRI study. Neuroimage 55: 1716-1727.

57. Mayer AR, Mannell MV, Ling J, Gasparovic C, Yeo RA (2011) Functional connectivity in mild traumatic brain injury. Hum Brain Mapp 32: 1825-1835

58. Shumskaya E, Andriessen TM, Norris DG, Vos PE (2012) Abnormal wholebrain functional networks in homogeneous acute mild traumatic brain injury. Neurology 79: 175-182.

59. Messe A, Caplain S, Pelegrini-Issac M, Blancho S, Levy R, et al. (2013) Specific and evolving resting-state network alterations in post-concussion syndrome following mild traumatic brain injury. PLOS One 8: e65470.

60. Han K, Mac Donald CL, Johnson AM, Barnes Y, Wierzechowski L, et al. (2014) Disrupted modular organization of resting-state cortical functional connectivity in U.S. military personnel following concussive 'mild' blast-related traumatic brain injury. Neuroimage 84: 76-96. 